\title{
A Sleep Education and Hypnotics Reduction Program for Hospitalized Patients at a General Hospital
}

\author{
Seockhoon Chung $\bowtie$, Soyoung Youn, Boram Park, Suyeon Lee, and Changnam Kim \\ Department of Psychiatry, Asan Medical Center, University of Ulsan College of Medicine, Seoul, Republic of Korea
}

\begin{abstract}
Objective We applied a program of sleep education and hypnotics reduction for inpatients (the i-sleep program). This study explored whether the i-sleep program is effective for reducing the prescription rate of sleeping pills to inpatients in a general hospital.

Methods We estimated the proportion of inpatients prescribed hypnotics at admission to and discharge from the hospital, excluding pediatric care units, before (2014) and after (2015) the program. In addition, we estimated the proportion of inpatients prescribed sleeping pills among all inpatients on the first day of each month of 2014 and 2015.

Results The proportion of inpatients prescribed hypnotics as discharge medication among inpatients who had been prescribed them at the time of admission decreased significantly, from $57.0 \%$ to $46.8 \%$, after the i-sleep program ( $\mathrm{RR}=0.82,95 \%$ CI: $0.79-0.86)$. The proportion of inpatients newly prescribed sleeping pills after admission to the hospital did not significantly decrease $(1.97 \%$ to $2.00 \%$; $\mathrm{RR}=1.01,95 \% \mathrm{CI}: 0.96-1.07)$. The mean prescription rate of sleeping pills per day was $8.18 \%$ in 2014 and $7.78 \%$ in 2015 .

Conclusion The i-sleep program reduced the proportion of inpatients who continued to take sleeping pills from admission until discharge, although it did't reduce the prescription rate per day.

Psychiatry Investig 2018;15(1):78-83
\end{abstract}

Key Words Hypnotics, Inpatients, Insomnia, Sleep.

\section{INTRODUCTION}

Hospitalized patients commonly suffer from insomnia during their stay at the hospital. ${ }^{1,2}$ Alarming noises, harsh lighting, and clinical procedures combined with patients' illnesses, pain, reduced physical mobility, and medication side effects disturb sleep. ${ }^{3-11}$ Insomnia itself can aggravate existing medical conditions, lower the pain threshold, and induce depression or anxiety, which can result in a vicious cycle. ${ }^{11-14} \mathrm{Al}-$ though various factors are known to influence inpatients' sleep, it is not easy to remove triggering factors. In clinical practice, physicians usually attempt to improve inpatients' sleep using sleeping pills.

Previous studies have indicated that inpatients more often use sleeping pills than the general population. ${ }^{15-17}$ We hypoth-

Received: November 27, 2016 Revised: February 22, 2017

Accepted: April 16, 2017 Available online: November 29, 2017

$\triangle$ Correspondence: Seockhoon Chung, MD, PhD

Department of Psychiatry, Asan Medical Center, University of Ulsan College of Medicine, 88 Olympic-ro 43-gil, Songpa-gu, Seoul 05505, Republic of Korea Tel: +82-2-3010-3411, Fax: +82-2-485-8381, E-mail: schung@amc.seoul.kr

(a) This is an Open Access article distributed under the terms of the Creative Commons Attribution Non-Commercial License (http://creativecommons.org/licenses/bync/4.0) which permits unrestricted non-commercial use, distribution, and reproduction in any medium, provided the original work is properly cited. esize that hospital stays increase the prescription rate for sleeping pills among inpatients, and we explored this rate at Asan Medical Center. ${ }^{18}$ We found that among 118,475 inpatients admitted in 2014, excluding patients in pediatric care units, 4,205 (3.54\%) were already taking sleeping pills at the time of admission. At the time of discharge, 2,396 (57.0\%) of these inpatients were still taking sleeping pills. In all, 2,256 inpatients began their prescription of sleeping pills post-admission and were discharged with sleeping pills as discharge medications. If the physician prescribed sleeping pills to inpatients during their hospital stay without any information about the discontinuation of the pills, patients tended to continue to take them for a long time. Thus, disseminating information about good sleep hygiene and sleeping-pill discontinuation to inpatients is recommended.

Researchers have developed various sleep programs and have reported the effectiveness of these programs for hospitalized patients. ${ }^{19-21}$ However, such programs usually focus on sleep-hygiene education or relaxation training for inpatients. We have also developed a sleep education and hypnotics reduction program for hospitalized patients, ${ }^{21}$ focused on sleep-hygiene education and relaxation training for inpatients. Preliminarily, we applied the program to inpatients in a psychi- 
atric open ward, and we found that the program decreased the prescription rates of sleeping pills. ${ }^{21}$ However, it is necessary to educate not only inpatients but also medical staff about sleep hygiene and how to prescribe sleeping pills to inpatients. We have updated our program accordingly ${ }^{22}$ and have applied it to all hospitalized patients regardless of their disease at Asan Medical Center.

The objective of this study is to explore whether the i-sleep program is effective to reduce the proportion of sleeping pills prescription rates among the hospitalized patients. For that, we investigated and compared the sleeping pills prescription rates in a general hospital before and after the sleep education and hypnotics reduction program.

\section{METHODS}

This is a prospective observational study. Data was obtained from Asan Medical Center (Seoul, Korea), the largest single hospital in Korea. Over 110,000 patients are admitted per year, and more than 2,100 inpatients stay per day on 2,704 licensed beds. The samples in this study were all inpatients excluding ones in the pediatric units in Asan Medical Center at 2014 and 2015. We did not obtain any personal private data of the patients. Data in this study was driven from clinical and research sources in the hospital, and an honest broker provided it to researchers after de-identification. The study protocol was approved by the Institutional Review Board of Asan Medical Center (2014-0886).

\section{Program of sleep-hygiene education and sleeping-pill reduction for hospitalized patients}

We developed a program of sleep-hygiene education and sleeping-pill reduction for hospitalized patients (the i-sleep program) during 2014. ${ }^{22}$ The program consists of two parts: system/environment improvement and staff education/promotion (Figure 1). Regarding the system/environment, our program provides such information to hospitalized patients through educational leaflets in the inpatient guidebook and educational video clips ${ }^{23}$ broadcast over the in-house broadcasting system. The video clips are also accessible anytime and anywhere on smartphones using the QR code. Regarding staff education/promotion, we believe that it is very important to educate medical staff about good sleep during hospitalization. Previously, the inpatient sleep program had been focused on education of inpatients. However, prescriptions for sleeping pills were usually ordered by residents, and sometimes pro re nata (PRN) orders were performed by nurses in the ward. Most physicians at the hospital do not know how to appropriately prescribe sleeping pills to inpatients. A sleep specialist repeatedly lectured doctors and nurses at the hospital on how to

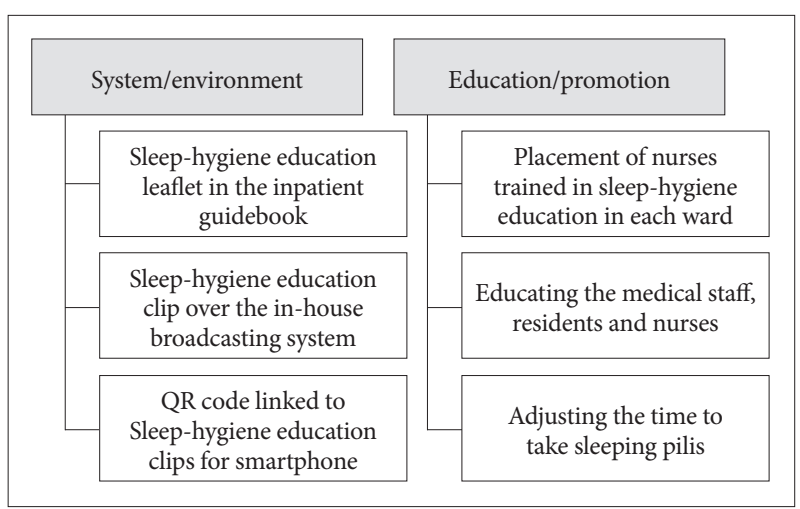

Figure 1. The sleep education and hypnotics reduction program at Asan Medical Center (the i-sleep program).

prescribe sleeping pills and how to help inpatients reduce or stop taking their pills. We placed nurses trained in sleep-hygiene education in each ward and had them educate inpatients on good sleep hygiene. We also educated medical staff, residents, and nurses about good sleep hygiene. We repeatedly promoted the i-sleep program to medical staff and nurses, and informed them that they would be able to acquire knowledge of appropriate prescription of sleeping pills through this program. The i-sleep program has been in effect at Asan Medical Center since January 1, 2015.

\section{Estimation of the use of sleeping pills in a general hospital}

We estimated the proportion of patients taking sleeping pills among hospitalized patients in two ways. First, we estimated the proportion of patients already taking sleeping pills at the time of admission and the proportion of patients given sleeping pills at the time of discharge from the hospital among all newly admitted patients during 2014 and 2015 (excluding patients in pediatric care units). Using these data, we explored how much the prescription rate of hypnotics changed during hospital stays. Second, we estimated the proportion of patients who were prescribed sleeping pills on the first day of each month of 2014 and 2015 to assess how many inpatients were taking sleeping pills per day at our hospital.

The sleeping pills we considered were zolpidem in its immediate-release (IR) or extended-release (ER) form, triazolam, benzodiazepines including clonazepam and bromazepam, and trazodone. We only considered a prescription as a sleeping pill when it was administered to patients in the evening with the prescription code hora somni (HS). If the medication was prescribed during the daytime, we did not consider it to be used as a sleeping pill.

\section{Statistical analysis}

Statistical analyses were performed with SPSS ver. 20.0 for 
Windows (IBM Corp., Armonk, NY, USA). The level of significance was defined as $\mathrm{p}<0.05$ in two-tailed tests for all analyses. Firstly, we estimated relative risk using the data of the proportion of inpatients who continued to take sleeping pills until discharge from the hospital at 2014 and 2015. And the Student's t-test was done using the proportion of sleeping pills prescription per day of 2014 and 2015.

\section{RESULTS}

A total of 118,475 patients (2014, before the i-sleep program) and 120,973 patients (2015, after the i-sleep program), excluding those in pediatric care units, were admitted to Asan Medical Center during the study period. In 2014, 4,205 (3.54\%) inpatients were already taking sleeping pills at the time of admission and 4,652 (3.92\%) inpatients were prescribed sleeping pills at the time of discharge (Table 1, Figure 2). In 2015, 4,584 (3.79\%) inpatients were already taking sleeping pills at admission, and 4,765 (3.93\%) were prescribed sleeping pills as discharge medications. The most frequently prescribed sleeping pills were zolpidem (IR or ER), benzodiazepine (bromazepam or clonazepam), triazolam, and trazodone in that order during 2014 and 2015 (Table 1).

Among the 4,205 inpatients already taking sleeping pills at the time of admission in 2014, 2,396 (57.0\%) were still taking pills at the time of discharge. Among the 4,584 inpatients who were already taking sleeping pills at the time of admission in 2015, 2,144 (46.8\%) were still taking them at discharge. These data indicate a significant decrease in the prescription rate after the i-sleep program ( $\mathrm{RR}=0.82,95 \% \mathrm{CI}$ : $0.79-0.86)$. Among all inpatients not prescribed sleeping pills at admission (11,4270 in 2014 and 116,389 in 2015), 2,256 (1.97\%) in 2014 and 2,325 (2.00\%) in 2015 were newly prescribed sleeping pills during their hospital stay ( $\mathrm{RR}=1.01,95 \% \mathrm{CI}$ : $0.96-1.07)$.

On the first day of each month of 2014, 7.3-10.0\% (mean $8.18 \%$ ) of inpatients were prescribed sleeping pills (Figure $3 \mathrm{~A}$ ). After the program, $6.8-9.0 \%$ (mean $7.78 \%$ ) of inpatients were prescribed the pills (Figure 3B). There were no significant changes in the mean proportion of inpatients prescribed sleeping pills on every first day of each month after the program $(\mathrm{p}=0.24)$. The mean proportion of inpatients taking one tablet of a sleeping pill was $80.3 \%$ in 2014 and $80.1 \%$ in 2015. The mean proportion of inpatients taking 2 kinds (or tablets) of sleeping pills was $15.4 \%$ in 2014 and $12.0 \%$ in 2015, and more than 3 kinds (or tablets) of sleeping pills was $4.3 \%$ in 2014 and $4.1 \%$ in 2015.

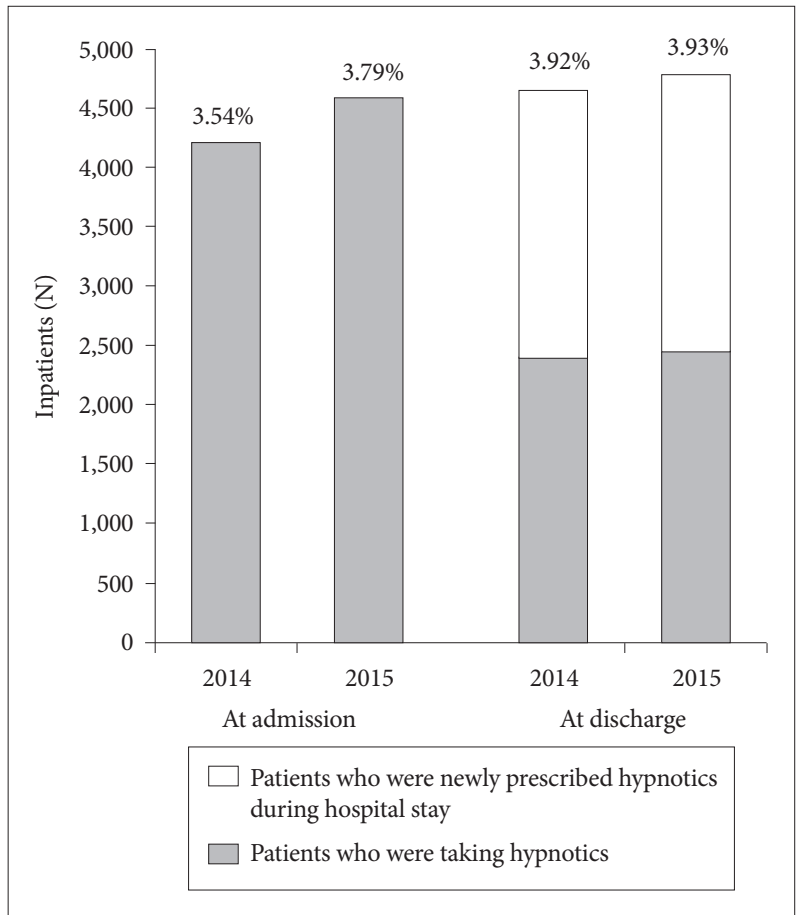

Figure 2. Proportion of patients prescribed hypnotics at time of admission or discharge among all patients admitted in 2014 (before the program) and 2015 (after the program). In 2014, 4,205 patients were taking sleeping pills at the time of admission, and 2,396 of these $(57.0 \%)$ were prescribed these pills as discharge medications. However, in 2015, 4,584 patients were taking sleeping pills at admission, and 2,144 of these $(46.8 \%)$ were prescribed these pills at discharge $(\mathrm{RR}=0.82,95 \% \mathrm{Cl}: 0.79-0.86)$. RR: relative risk, $\mathrm{Cl}$ : confidence interval.

Table 1. Inpatients prescribed sleeping pills at the time of admission or discharge before (2014) and after (2015) the program

\begin{tabular}{|c|c|c|c|c|c|c|}
\hline & \multicolumn{4}{|c|}{1 tablet } & \multirow[b]{2}{*}{2 tablets } & \multirow[b]{2}{*}{$\geq 3$ tablet } \\
\hline & $\begin{array}{l}\text { Zolpidem } \\
\text { IR or CR }\end{array}$ & Triazolam & $\begin{array}{c}\text { Clonazepam or } \\
\text { bromazepam }\end{array}$ & Trazodone & & \\
\hline \multicolumn{7}{|l|}{ Before program (2014) } \\
\hline At time of admission $(\mathrm{N}=4,205,3.54 \%)$ & $2,394(56.9)$ & $209(5.0)$ & $812(19.3)$ & $188(4.5)$ & $487(11.6)$ & $115(2.7)$ \\
\hline At time of discharge $(\mathrm{N}=4,652,3.92 \%)$ & $2,665(57.3)$ & $257(5.5)$ & $865(18.6)$ & $220(4.7)$ & $518(11.1)$ & $127(2.7)$ \\
\hline \multicolumn{7}{|l|}{ After program (2015) } \\
\hline At time of admission $(\mathrm{N}=4,584,3.79 \%)$ & $2,569(56.0)$ & $192(4.2)$ & $983(21.4)$ & $165(6.4)$ & $531(11.6)$ & $144(3.1)$ \\
\hline At time of discharge $(\mathrm{N}=4,765,3.93 \%)$ & $2,616(54.9)$ & $263(5.5)$ & $1,052(22.1)$ & $196(4.1)$ & $523(11.0)$ & $125(2.6)$ \\
\hline
\end{tabular}

IR: immediate-release, CR: extended-release 


\section{DISCUSSION}

We explored whether the i-sleep program at Asan Medical Center was effective for reducing the prescription of sleeping pills for inpatients. We found that the proportion of inpatients who continued to take sleeping pills until discharge from the hospital was reduced after the implementation of this program ( $R R=0.82,95 \% \mathrm{CI}: 0.79-0.86)$. Although these results do not reveal the direct impact of the i-sleep program on inpatient sleep, we believe that the long-term application of this program will help improve inpatient sleep and reduce the prescription rate of sleeping pills.

Numerous sleep programs have been applied to inpatients. ${ }^{19-21,24,25}$ Our inpatient sleep program consisted of two parts: system/environment improvement and staff education/promotion (Figure 1). Our program provides such information to hospitalized patients through educational leaflets in the inpatient guidebook and educational video clips ${ }^{23}$ broadcast over the in-house broadcasting system. And also we educated medical staff, residents, and nurses about good sleep
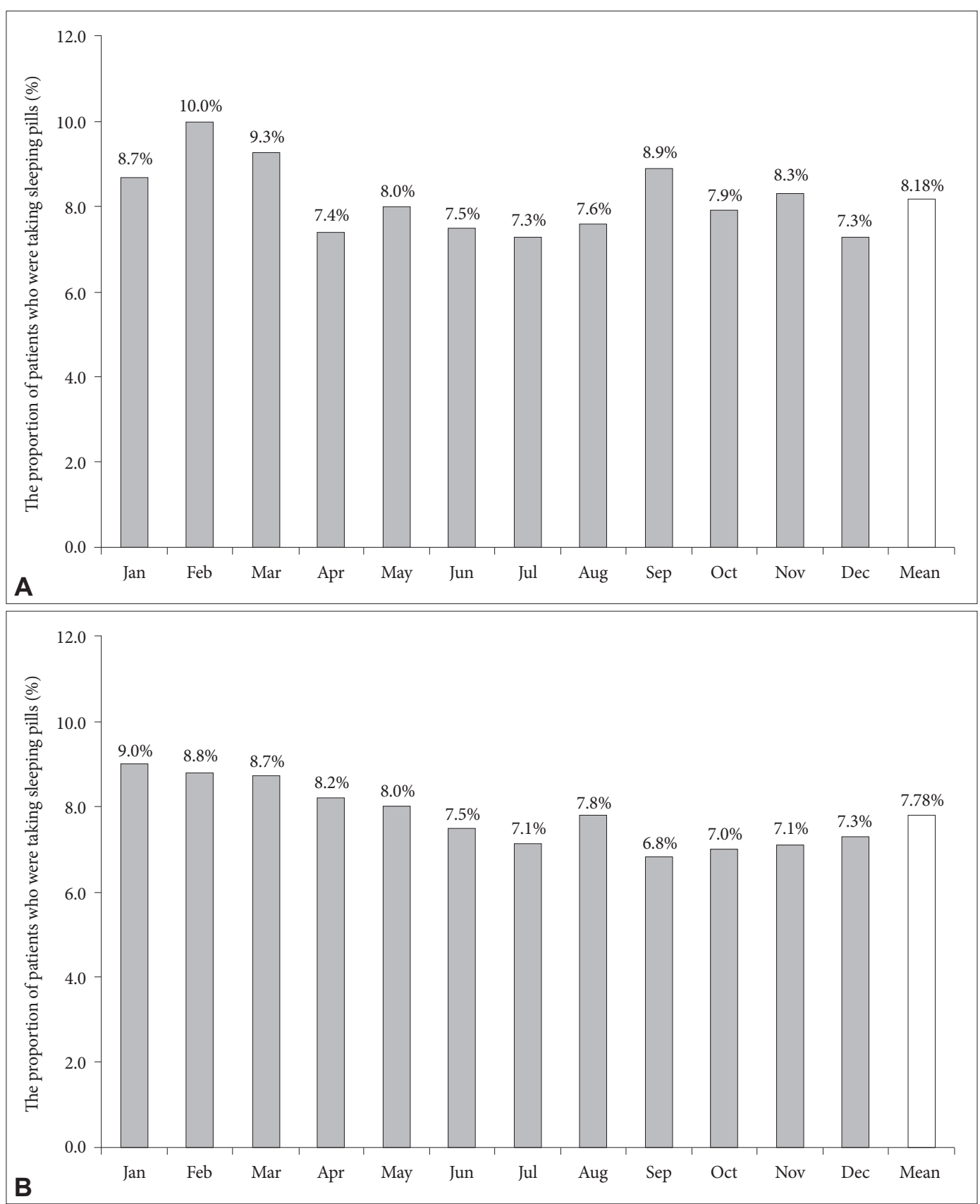

Figure 3. Proportion of patients taking hypnotics among all inpatients when assessed on the first day of each month in (A) 2014 (before the program) and (B) 2015 (after the program). 
hygiene during the study. We repeatedly promoted the i-sleep program to medical staff and nurses, and informed them that they would be able to acquire knowledge of appropriate prescription of sleeping pills through this program.

However, the proportion of inpatients newly prescribed sleeping pills during their hospital stay was not significantly lower after the program ( $\mathrm{RR}=1.01,95 \% \mathrm{CI}$ : 0.96-1.07): 7.3$10.0 \%$ (mean $8.18 \%$ ) of inpatients in 2014 and $6.8-9.0 \%$ (mean $7.78 \%$ ) of inpatients in 2015. It was difficult not to prescribe sleeping pills to inpatients with acute illnesses who could not sleep well. Inpatients who suffer from immobility, medical or neurological disease, pain, or mental disorders, including depression or anxiety disorders, usually spend most of their time in bed during the day, ${ }^{3,9-13}$ and this excessive amount of time in bed may disturb their sleep at night. Although medical staff usually encourage inpatients to move around and not to lay down in bed during the day, it is not easy to apply only sleep-hygiene education without sleeping pills to inpatients when they are in a phase of acute illness. The Korean style of housing patients in the hospital might also play a role. Six-bed rooms, which are very popular in all hospitals in South Korea, can be noisy, crowded, and impersonal environments compared to single-bed rooms. ${ }^{26,27}$ In six-bed rooms, it is not easy to establish a sleep-wake cycle individually, as the lights tend to be turned off in the evening at a fixed time around 09:30-10:00 pm. Cognitive-behavioral therapy (CBT) for insomnia could help reduce sleep disturbances for inpatients. Indeed, a previous study reported that patients were willing to attempt non-pharmacological treatment for insomnia during their hospital stays. ${ }^{28}$ However, it is not easy to use CBT for inpatients with different sleep-wake cycles in the same room.

We should also adjust the administration time of patients' sleeping pills so they do not take the pills too early. The administration time may also affect their sleep quality. Physicians usually order inpatients to take hypnotics hora somni which generally means 09:00 pm in most hospitals in South Korea. In terms of the sleep-wake cycle, 09:00 pm is somewhat early for inpatients that usually wake up at 6 or 7 in the morning. We have previously reported an association between the administration time of sleeping pills and patient satisfaction with sleeping pills. ${ }^{29,30}$ Patients who were satisfied with their pills tended to take benzodiazepine or non-benzodiazepine gamma-aminobutyric acid (GABA) agonists around 11:11 pm, where patients who were not satisfied tended to take their sleeping pills around 9:16 pm. Based on these results, sleeping pills may be less effective when taken earlier. However, the concept of individualizing or optimizing a prescription for sleeping pills is not popular among physicians unfamiliar with sleep medicine. Thus, education and systemic evolution are necessary for sleep hygiene, and proper prescription of sleeping pills is needed to decrease the proportion of inpatients taking such pills.

This study had several limitations of note. First, we estimated the prescription rate of sleeping pills at two time points: admission and discharge. Thus, it was unclear whether inpatients were transiently prescribed sleeping pills and how frequently the patients took pills during their hospital stay. Second, it was unclear whether the decrease in the prescription rate from 2014 to 2015 was obtained directly from the i-sleep program. More long-term follow-up analysis is needed to confirm the effects of the i-sleep program. Third, we defined hypnotics as triazolam, non-benzodiazepine GABA agonists (zolpidem and zolpidem CR, the only available z-drug in South Korea), trazodone, and benzodiazepines such as bromazepam and clonazepam. However, we cannot determine whether these medications were ordered by doctors exclusively for insomnia or for other purposes. Benzodiazepine can also be prescribed for anxiety disorders, movement disorders, or specific sleep disorders such as restless legs syndrome, periodic limb movement disorder, non-REM parasomnias, and REM sleep behavior disorder. Finally, other medications, such as several tricyclic antidepressants, antipsychotics, and prolonged-release melatonin, were not considered in the present study. Finally, we could not estimate the exact dosage of each sleeping pills prescribed to inpatients. More detailed estimation using hospital big data is needed in a further study. However, despite these limitations, our data indicate that the i-sleep program reduced the prescription rate of sleeping pills at our hospital.

\section{REFERENCES}

1. Shapiro CM, Devins GM, Hussain MR. ABC of sleep disorders. Sleep problems in patients with medical illness. BMJ 1993;306:1532-1535.

2. Henoch I, Sawatzky R, Falk H, Fridh I, Jakobsson Ung E, et al. Symptom distress profiles in hospitalized patients in Sweden: a cross-sectional study. Res Nurs Health 2014;37:512-523.

3. Venkateshiah SB, Collop NA. Sleep and sleep disorders in the hospital. Chest 2012;141:1337-1345.

4. Meyer TJ, Eveloff SE, Bauer MS, Schwartz WA, Hill NS, Millman RP. Adverse environmental conditions in the respiratory and medical ICU settings. Chest 1994;105:1211-1216.

5. Celik S, Oztekin D, Akyolcu N, Issever H. Sleep disturbance: the patient care activities applied at the night shift in the intensive care unit. J Clin Nurs 2005;14:102-106.

6. Tamburri LM, DiBrienza R, Zozula R, Redeker NS. Nocturnal care interactions with patients in critical care units. Am J Crit Care 2004;13: 102-112; quiz 114-115.

7. Konkani A, Oakley B. Noise in hospital intensive care units--a critical review of a critical topic. J Crit Care 2012;27:522.

8. Park MJ, Yoo JH, Cho BW, Kim KT, Jeong WC, Ha M. Noise in hospital rooms and sleep disturbance in hospitalized medical patients. Environ Health Toxicol 2014;29:e2014006.

9. Young JS, Bourgeois JA, Hilty DM, Hardin KA. Sleep in hospitalized medical patients, part 1: factors affecting sleep. J Hosp Med 2008;3:473- 
482

10. Reid E. Factors affecting how patients sleep in the hospital environment. Br J Nurs 2001;10:912-915.

11. Bernhofer EI, Higgins PA, Daly BJ, Burant CJ, Hornick TR. Hospital lighting and its association with sleep, mood and pain in medical inpatients. J Adv Nurs 2014;70:1164-1173.

12. Tang NK, Salkovskis PM, Hodges A, Wright KJ, Hanna M, Hester J. Effects of mood on pain responses and pain tolerance: an experimental study in chronic back pain patients. Pain 2008;138:392-401.

13. Villemure C, Bushnell MC. Mood influences supraspinal pain processing separately from attention. J Neurosci 2009;29:705-715.

14. Arora VM, Chang KL, Fazal AZ, Staisiunas PG, Meltzer DO, Zee PC, et al. Objective sleep duration and quality in hospitalized older adults: associations with blood pressure and mood. J Am Geriatr Soc 2011;59: 2185-2186.

15. Ohayon MM, Lader MH. Use of psychotropic medication in the general population of France, Germany, Italy, and the United Kingdom. J Clin Psychiatry 2002;63:817-825.

16. Bertisch SM, Herzig SJ, Winkelman JW, Buettner C. National use of prescription medications for insomnia: NHANES 1999-2010. Sleep 2014; 37:343-349.

17. Chung S, Park B, Yi K, Lee J. Pattern of hypnotic drug prescription in South Korea: health insurance review and assessment service national patients sample. Sleep Med Res 2013;4:51-55.

18. Youn S, Hann CC, Park B, Lee S, Kim C, Yi K, et al. The sleeping pill prescription rate for inpatients at a genetal hospital. Sleep Med Res 2016;7:33-38.

19. Lareau R, Benson L, Watcharotone K, Manguba G. Examining the feasibility of implementing specific nursing interventions to promote sleep in hospitalized elderly patients. Geriatr Nurs 2008;29:197-206.

20. McDowell JA, Mion LC, Lydon TJ, Inouye SK. A nonpharmacologic sleep protocol for hospitalized older patients. J Am Geriatr Soc 1998;46: 700-705.

21. Youn S, Son JI, Chung S. Preliminary results of sleep pill reduction program for psychiatric inpatients. Kor J Sleep Med 2010;12:19-22.

22. Youn S, Yi K, Park B, Lee S, Jung YS, Choi JS, et al. Development of sleep-hygiene education and sleeping pills reduction program for hospitalized patients. Kor J Sleep Med 2015;17:33-42.

23. A good sleep hygiene during the hospital stay. Available at: https://www. youtube.com/watch?v=8C-OJqF7MXM. Accessed November 1, 2016.

24. De Sousa IC, Araujo JF, De Azevedo CVM. The effect of a sleep hygiene education program on the sleep-wake cycle of Brazilian adolescent students. Sleep Biol Rhythms 2007;5:251-258.

25. Nishinoue N, Takano T, Kaku A, Eto R, Kato N, Ono Y, et al. Effects of sleep hygiene education and behavioral therapy on sleep quality of whitecollar workers: a randomized controlled trial. Ind Health 2012;50:123131.

26. Persson E, Anderberg P, Ekwall AK. A room of one's own--Being cared for in a hospital with a single-bed room design. Scand J Caring Sci 2015; 29:340-346.

27. van de Glind I, de Roode S, Goossensen A. Do patients in hospitals benefit from single rooms? A literature review. Health Policy 2007;84:153161.

28. Azad N, Byszewski A, Sarazin FF, McLean W, Koziarz P. Hospitalized patients' preference in the treatment of insomnia: pharmacological versus non-pharmacological. Can J Clin Pharmacol 2003;10:89-92.

29. Chung S, Youn S, Yi K, Park B, Lee S. Sleeping pill administration time and patient subjective satisfaction. J Clin Sleep Med 2016;12:57-62.

30. Youn S, Hann CC, Park B, Lee S, Yi K, Chung S. The effects of the new guidance 'take your sleeping pills $7 \mathrm{~h}$ before your wake-up time': a pilot study. Sleep Biol Rhythms 2016;14:397-404. 